\title{
Ensamble de coleópteros epigeos ribereños del Humedal de Batuco (Región Metropolitana, Chile)
}

\section{Assemblage of riparian epigeal coleopteran of the Batuco Wetland (Metropolitan Region, Chile)}

\section{Carolain Badaracco ${ }^{1}$, Amanda Huerta $^{1, *}$ \& Álvaro Palma²}

1Laboratorio de Entomología Forestal, Departamento de Silvicultura y Conservación de la Naturaleza, Facultad de Ciencias Forestales y de la Conservación de la Naturaleza, Universidad de Chile, Casilla 9206, Santiago, Chile.

'Laboratorio de Criminalística Central, Policía de Investigaciones de Chile, Carlos Silva Vildósola N9783, La Reina, Santiago, Chile.

*E-mail: ahuerta@uchile.cl

\section{RESUMEN}

El Humedal de Batuco (Región Metropolitana, Chile) se sitúa en uno de los sitios prioritarios de conservación de biodiversidad de la zona Central de Chile. Dada la escasa información acerca de su fauna entomológica, se estudió en este sitio el ensamble de coleópteros epigeos ribereños en dos temporadas: primavera (2015) y otoño (2016). Alrededor del borde del cuerpo de agua se seleccionaron tres tipos de vegetación (TV) representativos de las especies vegetales dominantes. En cada uno de estos TV, se instaló al azar cinco trampas de intercepción (pitfall) a una distancia mínima de $5 \mathrm{~m}$ desde el borde por temporada $(n=30)$ para la captura de los coleópteros. Posterior a su identificación se estimó la abundancia y riqueza de los coleópteros y de los gremios tróficos asociados: fitófagos, depredadores y saprófagos. Se aplicaron pruebas no paramétricas de Kruskal-Wallis y de Mann-Whitney, para determinar las diferencias estadísticas en las variables evaluadas. El ensamble de coleópteros epigeos estuvo conformado por seis familias, 14 géneros y 20 especies (12 de ellas nativas). La abundancia y riqueza de los coleópteros epigeos fue significativamente superior en primavera en todos los TV evaluados. En los gremios se produjeron diferencias en la abundancia en los depredadores y saprófagos en primavera, en tanto que por temporada en los depredadores se observaron diferencias en cada TV. La información obtenida constituye el primer registro de entomofauna epigea del borde del humedal, y puede servir de base para investigaciones futuras sobre conservación en este sitio prioritario.

Palabras clave: abundancia, Carabidae, riqueza.

\section{ABSTRACT}

The Batuco Wetland (Metropolitan Region, Chile) is located in one of the priority sites for biodiversity conservation in Central Chile. Given the scarce information about its entomological fauna, the assemblage of riparian epigeal beetles was studied in two seasons: spring (2015) and fall (2016). Around the edge of wetland water body, three types of vegetation (TV) representatives of the dominant plant species were selected. In each of these TV, five interception traps (pitfalls) were randomly installed at a minimum distance of $5 \mathrm{~m}$ from the edge per season $(n=30)$ to capture of the beetles. After its identification, the abundance and richness of the coleopterans and associated trophic guilds were estimated: phytophagous, predatory and saprophagous. Nonparametric tests of Kruskal-Wallis and Mann-Whitney were applied to determine the statistical differences between the evaluated variables. The epigeal coleopteran assembly 
was made up of six families, 14 genera and 20 species (12 of them native). Epigeal coleopteran abundance and richness were significantly higher in spring in all TV evaluated. In the guilds there were differences in abundance in predators and saprophagous in spring, while by season in predators' differences were observed in each TV. The information obtained constitutes the first record of epigeal entomofauna in the edge of the wetland and may serve as a basis for future research aimed at conserving this priority site.

Keywords: abundance, Carabidae, richness.

\section{INTRODUCCIÓN}

En las últimas décadas las áreas con vegetación han ido cambiando drásticamente debido a la modificación de los usos del suelo, convirtiéndose las superficies de bosques y matorrales en terrenos agrícolas, praderas y zonas urbanas, con la consecuente pérdida de la biodiversidad (Sala et al. 2000).

Los factores que inciden en la degradación y destrucción de un ecosistema han perturbado a la biota en diversos niveles. Algunos taxa de insectos se han visto afectados en su conservación a nivel mundial, debido al calentamiento global, la sobreexplotación, introducción de nuevas especies, entre otros factores (Briones et al. 2012; Barba-Álvarez et al. 2013; Jerez et al. 2015). La estructura y abundancia de las poblaciones de insectos están muy relacionadas con la disponibilidad de recursos (Castiglioni et al. 2017). Así, la calidad y estructura de la vegetación influyen en los patrones de distribución espacial de los insectos mediante la disponibilidad de su alimento (Castro et al. 2012). No obstante, aún son considerados como los animales más representativos en cuanto a biomasa, variedad genética e interacciones bióticas en los ecosistemas terrestres; además, cumplen con una gama de funciones muy amplia, actuando como polinizadores, descomponedores, parásitos y depredadores (Vásquez et al. 2006; Bautista et al. 2011). De las especies de insectos, cerca del $40 \%$ corresponden a coleópteros, considerados sujetos idóneos como bioindicadores, por su facilidad de muestreo, su abundancia, riqueza de especies, y sobre todo debido a su alta sensibilidad frente a perturbaciones (Roig-Juñent \& Domínguez 2001; Vásquez et al. 2006). Los coleópteros en Chile están representados por 102 familias, 1.348 géneros y más de 4.454 especies (MMA 2018).

La ecorregión del matorral y bosque esclerófilo $\left(30^{\circ}\right.$ a $36^{\circ} \mathrm{S}$ ) de Chile ha sido una de las zonas más afectadas por la intensa presión antrópica, con una concentración cercana al $70 \%$ de la población, lo que ha dificultado su protección a través del Sistema Nacional de Áreas Silvestres Protegidas del Estado (SNASPE) (Donoso 1993). Por su parte, el tipo forestal esclerófilo ocupa 345.089 ha que representan el 2,6\% de la superficie nacional de bosque nativo, donde sólo el $2 \%$ de ésta, se encuentra resguardada por el SNASPE (Martínez et al. 2011). Cabe destacar que dicha área está clasificada como uno de los sitios prioritarios para la conservación de la biodiversidad a nivel mundial, dada la gran diversidad de especies, grado de endemismo alto, junto a la amenaza antrópica (Myers et al. 2000). Además, forma parte de uno de los 34 puntos calientes del planeta (Mittermeier et al. 2004).

Dentro de los ecosistemas más frágiles se distinguen los humedales que conforman zonas de transición terrestreacuáticas, incluyendo también las áreas costeras y ribereñas adyacentes (CONAF 2010). Uno de los humedales más representativo y de acceso fácil es el Humedal de Batuco (Comuna de Lampa, Región Metropolitana), situado en la depresión intermedia de la cuenca de Santiago (Fig. 1). En este humedal se ha evidenciado que recibe aportes hídricos contaminados que llegan a los depósitos subterráneos por infiltración directa, junto al impacto antrópico por la agricultura y la ganadería que se practica en esos sectores (Del Campo et al. 2005). De acuerdo con la Estrategia para la Conservación de la Biodiversidad corresponde al "Sitio Prioritario N ${ }^{\circ} 6^{\prime}$, cuya superficie total es de 14.778 ha (CONAMA 2004). Este humedal se considera de conservación biológica, como área de protección oficial para efectos del Sistema de Evaluación de Impacto Ambiental, así como también de interés turístico, donde está prohibida la caza (CONAMA 2004; 2010). Actualmente se encuentra en la fase de declaración de Santuario de la Naturaleza (MMA 2021). No existen antecedentes sobre la entomofauna albergada en este humedal, por lo que se planteó como objetivo estudiar el ensamble de coleópteros epigeos ribereños y explorar la variación estacional de su abundancia y riqueza, de acuerdo con la composición florística. 


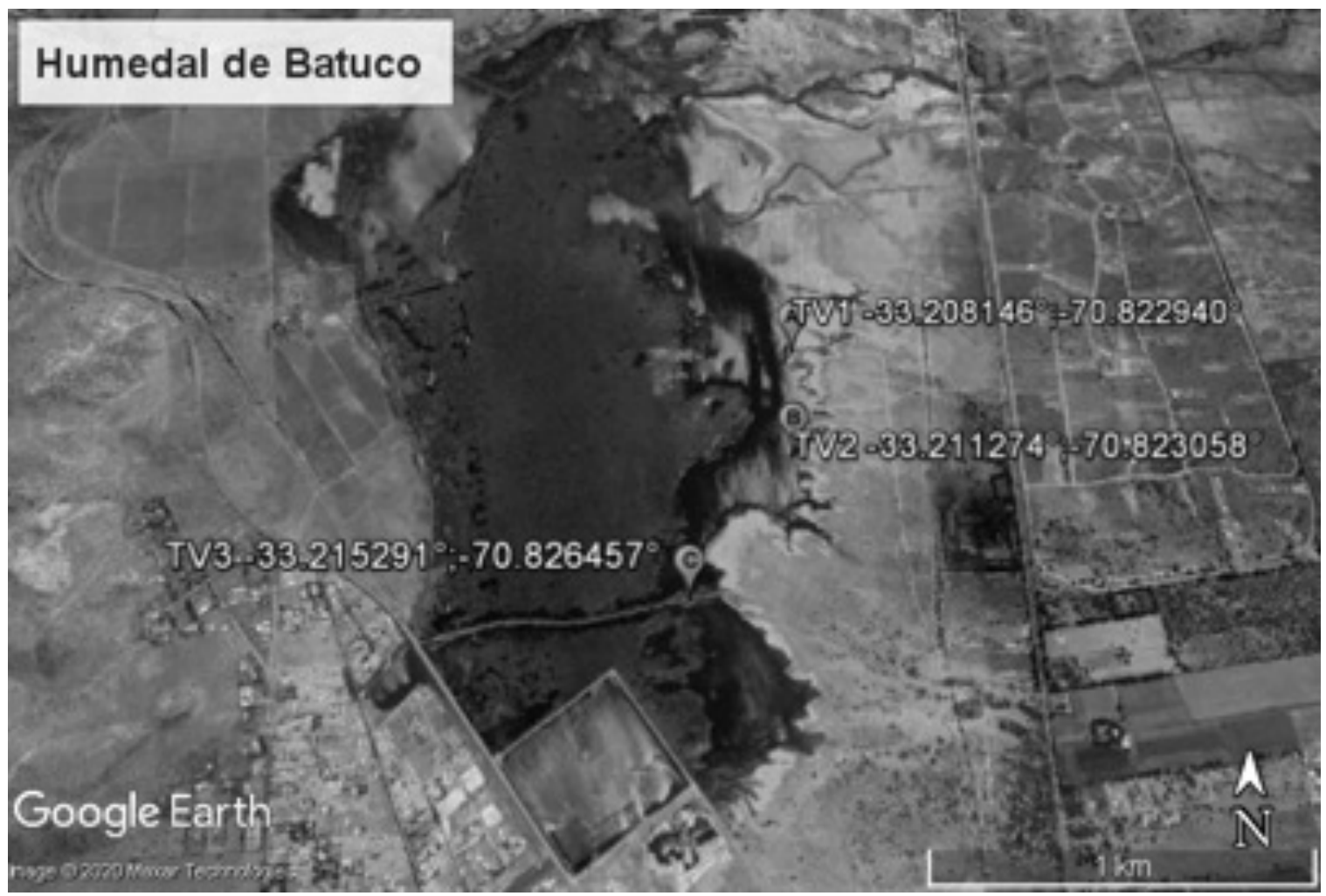

Figura 1. Mapa del Humedad de Batuco (Comuna de Lampa, Región Metropolitana) con sus tipos de vegetación (TV). / Map of Batuco Wetland (Community of Lampa, Metropolitan Region) with its types of vegetation (TV).

\section{MATERIALES Y MÉTODOS}

\section{ÁreA DE ESTUDIO}

El estudio se realizó en el Humedal de Batuco (3312"40' S, 7049"23' O), ubicado al norponiente de Santiago, Comuna de Lampa, Provincia de Chacabuco (Región Metropolitana, Chile). El área presenta condiciones climáticas mediterráneas, con inviernos fríos y lluviosos, y veranos cálidos y secos. La precipitación total anual es de $308 \mathrm{~mm}$ con una temporada seca de ocho meses. La temperatura máxima se registra en enero con una media de $31^{\circ} \mathrm{C}$, mientras que la mínima ocurre en julio con una media de $2,8^{\circ} \mathrm{C}$ (Uribe et al. 2012). Según la clasificación vegetacional de Luebert \& Pliscoff (2006), el humedal se inserta en la región del matorral y bosque esclerófilo. Alrededor del borde del humedal se distinguieron tres tipos de vegetación (TV) (Fig. 1), conformadas por vegetación heterogénea, dominada por las siguientes especies: TV1: Cressa truxillensis Kunth (Convolvulaceae) y Frankenia salina (Molina) I.M. Johnst. (Frankeniaceae); TV2: Chenopodium glaucum L. (Chenopodiaceae), Distichlis spicata (L.) Greene (Poaceae), Proustia cuneifolia D. Don (Asteraceae) y
F. salina, y TV3: Eleocharis macrostachya Britton (Cyperaceae), Scirpus californicus (C.A. Mey.) Steudel (Cyperaceae) y Typha angustifolia L. (Typhaceae). Cada TV tuvo un distanciamiento entre sí que fluctuó entre 300 a 1000 m.

\section{MÉTOdo de MUESTReo}

Se usaron trampas de intercepción (pitfall traps) para la captura de coleópteros epigeos, consistentes en contenedores plásticos con capacidad de $1 \mathrm{~L}$, siendo vertidos con $250 \mathrm{~mL}$ de etanol $\left(96^{\circ}\right)$ al $75 \%$ para preservar los especímenes y 500 $\mathrm{mL}$ de agua destilada con trazas de detergente para reducir la tensión superficial. En cada TV se instalaron al azar cinco trampas de intercepción a ras de suelo (Alvarado-Orellana et al. 2018), distanciadas entre sí por al menos $5 \mathrm{~m}$ desde la ribera del cuerpo de agua y por $10 \mathrm{~m}$ entre sí para evitar el traslape del radio de captura (5 m) (Buddle \& Hammond 2003; Cepeda-Pizarro et al. 2005). Esta actividad se hizo en primavera de 2015 y otoño de 2016, quedando instaladas las trampas durante 15 días (Briones \& Jerez 2007), y luego se procedió al retiro del material capturado, el que fue conservado en etanol al 70\% hasta su identificación. 


\section{IDENTIFICACIÓN DE LOS COLEÓPTEROS EPIGEOS}

Los coleópteros epigeos colectados se identificaron taxonómicamente mediante el uso de claves (Werner 1974; Morrone 1993; Artigas 1994; Roig-Juñent \& Domínguez 2001) y lupa estereoscópica, así como también se compararon morfológicamente con ejemplares de las colecciones entomológicas del Museo de Entomología Forestal de la Universidad de Chile (MEF, Curadora: Amanda Huerta) y colección de insectos del Museo Nacional de Historia Natural (MNHN, Curadora: Fresia Rojas), ambos en Santiago de Chile. Posteriormente mediante información bibliográfica cada especie identificada se clasificó conforme a su gremio trófico: fitófago, depredador y saprófago (Elgueta \& Arriagada 1989; Artigas 1994).

\section{Procesamiento de LA INFORMACIÓN}

Se determinó la abundancia (número de individuos/ha) de cada especie por TV y temporada. Posteriormente se calculó la abundancia (número promedio de individuos/ha), la riqueza (número promedio de especies/ha) de los coleópteros epigeos, y la abundancia (número promedio de individuos/ ha) de los gremios tróficos, con su respectivo error estándar, para cada TV y temporada (Moreno 2001). Para cada una de estas variables se comprobaron los supuestos de normalidad y homocedasticidad. Se utilizó la prueba no paramétrica de Kruskal-Wallis $(\mathrm{H})$ para comprobar las diferencias estadísticas entre TV y la prueba de Mann-Whitney (U) para identificar las diferencias entre temporadas. Ambas pruebas se realizaron con el programa estadístico InfoStat, versión 2011 (InfoStat 2011).

\section{RESULTADOS}

\section{COMPOSICIÓN TAXONÓMICA DE LOS COLEÓPTEROS EPIGEOS}

El ensamble de coleópteros epigeos de la ribera del Humedad de Batuco estuvo conformado por un total de 2.061 individuos distribuidos en seis familias, 14 géneros y 20 especies (de las cuales 12 fueron nativas), con 1.761 individuos en primavera y 300 en otoño (Tabla 1).

En cuanto a la abundancia de las familias de coleópteros epigeos, en primavera se registró un mayor número de individuos de la familia Carabidae (92,9\%) $(n=1.636)$, seguidas en menor proporción por Scarabaeidae (3,4\%) $(n=60)$, Anthicidae $(3,1 \%)(n=55)$, Elateridae $(0,3 \%)(n=5)$ y Heteroceridae $(0,3 \%)(n=5)$; mientras que en otoño sólo se detectaron dos familias, Carabidae $(81,7 \%)(n=245)$, la más abundante, y Curculionidae $(18,3 \%)(n=55)$ (Tabla 1).

Con respecto a la composición de especies se detectaron 18 especies en primavera, siendo los carábidos más abundantes, tales como: Pachymorphus lucidus (Curtis) ( $n=55)$, Bembidion (Nothapus) sp. ( $n=55)$ y Metius femoratus (Dejean) $(n=55)$. En cambio, en otoño se identificaron ocho especies, donde las más abundantes fueron Hyperoides subcinctus (Boheman) (Curculionidae) ( $n=55)$, Pachymorphus striatulus (Fabricius) (Carabidae) y Acupalpus sp. (Carabidae) ( $n=55)$ (Tabla 1).

Algunas especies fueron exclusivas de primavera, tal es el caso de Anthicus floralis (L.), Anthicus sp., Aphodius pseudolividus Balthasar,Argutoridiuschilensis(Dejean), Bembidion anthracium, Bembidion sp., Deromecus filicornis (Solier), Heterocerus sp., Metius femoratus (Dejean), Mimodromites cyaneus (Dejean), Parhypates sp. y Tetragonoderus viridis (Dejean). En cambio en otoño, sólo se detectó a Mimodromites nigrotestaceus (Solier) y Hyperoides subcinctus. Tras el análisis de la información obtenida se constató que la mayor abundancia y riqueza fue superior durante primavera (Tabla 1 ).

VARIACIÓN ESTACIONAL EN LA ABUNDANCIA Y RIQUEZA DE LOS COLEÓPTEROS EPIGEOS

La variación de la abundancia de coleópteros epigeos fue significativa en primavera $(H=12,50 ; p<0,01)$ entre los TV evaluados, siendo mayor en el TV3 (más del doble que en el TV 1), seguida del TV2 (1,6 veces); mientras que en otoño no se observaron diferencias significativas entre los $\mathrm{TV}(\mathrm{H}=$ $2,8 ; \mathrm{p}<0,05)$ estudiados. Al comparar entre temporadas, las variaciones de la abundancia fueron significativamente superiores en primavera: TV1 (triple que el de otoño) ( $U=$ 2,03; $p<0,05)$, TV2) (seis veces superior) $(U=2,02 ; p<0,05)$ y en el TV3 (diez veces) $(U=2,03 ; p<0,05)$. Cabe indicar que la mayor abundancia obtenida en primavera en el TV3 se debió en gran parte a una única especie, Pachymorphus lucidus (Curtis), siendo casi el triple de la siguiente abundancia más alta, obtenida con el también carábido Metius femoratus (Tabla 2).

La oscilación de la riqueza de coleópteros epigeos en primavera también fue significativa $(H=5,80 ; p<0,05)$, aunque menos marcadas entre los TV, siendo mayor en el TV2. Al igual que en el caso de la abundancia, en otoño no se detectaron diferencias significativas entre los TV $(H=1,0 ; p<0,05)$. Entre temporadas, la riqueza fue más elevada en primavera en el TV2 (U= 2,03; $p<0,05)$ (Tabla 2).

VARIACIÓN ESTACIONAL EN LA ABUNDANCIA DE LOS GREMIOS TRÓFICOS La abundancia en el gremio de los fitófagos según TV no presentó diferencias significativas en primavera $(H=5,10$; $p<0,05)$ ni en otoño $(H=2,71 ; p<0,05)$. En tanto que entre temporadas sólo se detectaron diferencias significativas $(\mathrm{U}=$ 1,76; $p<0,05$ ) en el TV2, siendo mayor en primavera (Tabla 2).

En la abundancia del gremio de los depredadores según TV se observaron variaciones significativas $(H=10,0 ; p<0,01)$ 
en primavera, siendo superior en el TV3 (más del doble que el observado en el TV1). No se detectaron diferencias en otoño $(H=1,83 ; p<0,05)$. Entre temporada se obtuvo diferencias significativas en la abundancia, siendo superiores en primavera en todos los TV: TV1 (cuádruple con respecto al otoño) $(U=2,02 ; p<0,05)$, TV2 (nueve veces) $(U=2,02$; $\mathrm{p}<0,05)$ y TV3 (doce veces) $(U=2,02 ; p<0,05)$ (Tabla 2$)$.

En los saprófagos, hubo diferencias significativas $(\mathrm{H}=$ 3,38; $p<0,05$ ) en los distintos TV en primavera, presentándose únicamente este grupo y en el TV3. Entre temporadas no hubo diferencias significativas en los TV evaluados (Tabla 2).

TABLA 1. Abundancia (número de individuos/ha) de especies de coleópteros epigeos ribereños por tipo de vegetación (TV) en primavera (P) y otoño (O) en el Humedal de Batuco (Santiago, Chile). ${ }^{*}$ Gremio Trófico D: Depredador, F: Fitófago, S: Saprófago. ${ }^{* *}$ N: Nativa. ${ }^{* * *}$ TV (Especies dominantes) TV1: Cressa truxillensis, Frankenia salina; TV2: Eleocharis macrostachya, Scirpus californicus, Typha angustifolia; TV3: Chenopodium glaucum, Distichlis spicata, Proustia cuneifolia. / Abundance (number of individuals/ha) of riparian epigeal beetle species by type of vegetation (TV) in spring (P) and autumn (O) in the Batuco Wetland (Santiago, Chile). " Trophic Guild D: Predator, F: Phytophage, s: Saprophage. ${ }^{* *}$ N: Native. ${ }^{* * *}$ TV (Dominant species): TV1: Cressa truxillensis, Frankenia salina; TV2: Eleocharis macrostachya, Scirpus californicus, Typha angustifolia; TV3: Chenopodium glaucum, Distichlis spicata, Proustia cuneifolia.

\begin{tabular}{|c|c|c|c|c|c|c|}
\hline & \multicolumn{6}{|c|}{ Tipo de vegetación ${ }^{* * *}$} \\
\hline & \multicolumn{2}{|c|}{ TV1 } & \multicolumn{2}{|r|}{ TV2 } & \multicolumn{2}{|c|}{ TV3 } \\
\hline & $\mathbf{P}$ & O & $\mathbf{P}$ & O & $\mathbf{P}$ & O \\
\hline \multicolumn{7}{|l|}{ Anthicidae } \\
\hline Anthicus floralis (Linnaeus, 1758) $\mathrm{F}$ & 0 & 0 & 26 & 0 & 0 & 0 \\
\hline Anthicus sp. ${ }^{\mathrm{F}}$ & 153 & 0 & 102 & 0 & 0 & 0 \\
\hline \multicolumn{7}{|l|}{ Carabidae } \\
\hline Acupalpus sp. ${ }^{\mathrm{D}}$ & 76 & 102 & 280 & 127 & 0 & 0 \\
\hline Argutoridius chilensis (Dejean, 1828) & 204 & 0 & 489 & 0 & 178 & 0 \\
\hline Bembidion anthracium (Germain, 1906) ${ }^{\mathrm{D}}$ & 433 & 0 & 153 & 0 & 0 & 0 \\
\hline Bembidion sp. ${ }^{\mathrm{D}}$ & 204 & 0 & 560 & 0 & 0 & 0 \\
\hline Metius femoratus (Dejean, 1828) D,N & 26 & 0 & 280 & 0 & 764 & 0 \\
\hline Mimodromites cyaneus (Dejean, 1831) & 127 & 0 & 0 & 0 & 0 & 0 \\
\hline Mimodromites nigrotestaceus (Solier, 1849) & 0 & 51 & 0 & 0 & 0 & 51 \\
\hline Pachymorphus lucidus (Curtis, 1839) & 204 & 51 & 433 & 51 & 2.215 & 0 \\
\hline Pachymorphus striatulus (Fabricius, 1775) & 127 & 102 & 229 & 76 & 178 & 76 \\
\hline Pachymorphus chalceus (Dejean, 1828) & 102 & 76 & 102 & 51 & 204 & 76 \\
\hline Pachymorphus sp. ${ }^{\mathrm{D}, \mathrm{N}}$ & 0 & 51 & 0 & 0 & 127 & 102 \\
\hline Parhypates sp. ${ }^{\mathrm{D}, \mathrm{N}}$ & 51 & 0 & 102 & 0 & 51 & 0 \\
\hline Pterostichus sp. ${ }^{\mathrm{F}}$ & 178 & 127 & 178 & 76 & 0 & 0 \\
\hline Tetragonoderus viridis (Dejean, 1831) ${ }^{\mathrm{D}, \mathrm{N}}$ & 0 & 0 & 76 & 0 & 0 & 0 \\
\hline \multicolumn{7}{|l|}{ Curculionidae } \\
\hline Hyperoides subcinctus (Boheman, 1842) & 0 & 51 & 0 & 102 & 0 & 127 \\
\hline \multicolumn{7}{|l|}{ Elateridae } \\
\hline Deromecus filicornis Solier, 1851:13F,N & 0 & 0 & 0 & 0 & 26 & 0 \\
\hline \multicolumn{7}{|l|}{ Heteroceridae } \\
\hline Heterocerus sp..$^{\mathrm{F}}$ & 0 & 0 & 26 & 0 & 0 & 0 \\
\hline \multicolumn{7}{|l|}{ Scarabaeidae } \\
\hline Aphodius pseudolividus Balthasar, 19415 & 0 & 0 & 0 & 0 & 306 & 0 \\
\hline
\end{tabular}


TABLA 2. Abundancia y riqueza de especies de coleópteros epigeos ribereños y abundancia de gremios tróficos asociados por tipo de vegetación (TV) en primavera (P) y otoño (O) en el Humedal de Batuco (Santiago, Chile). H: Prueba de Kruskal-Wallis. U: Prueba de

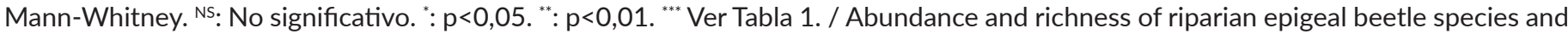
abundance of trophic guilds associated by type of vegetation (TV) in spring (P) and autumn (O) in the Batuco Wetland (Santiago, Chile). H: Kruskal-Wallis test. U: Mann-Whitney test. NS: Not significant. ${ }^{*}: \mathrm{p}<0.05$. $^{* *}$ : $\mathrm{p}<0.01$. $^{* *}$ See Table 1.

\begin{tabular}{|c|c|c|c|c|c|}
\hline & & & & & \\
\hline & & \multicolumn{4}{|c|}{ Tipo de vegetación ${ }^{* * *}$} \\
\hline & & TV1 & TV2 & TV3 & $\mathrm{H}$ \\
\hline \multirow{3}{*}{$\mathrm{N}^{\circ}$ promedio \pm error estándar de individuos/ha } & $\mathbf{P}$ & $1.884 \pm 94$ & $3.030 \pm 226$ & $4.049 \pm 271$ & $12,50^{* *}$ \\
\hline & 0 & $611 \pm 84$ & $484 \pm 62$ & $433 \pm 51$ & $2,80^{\mathrm{NS}}$ \\
\hline & U & $2,03^{*}$ & $2,02^{*}$ & $2,03^{*}$ & \\
\hline \multirow{3}{*}{$\mathrm{N}^{\circ}$ promedio \pm error estándar de especies/ha } & $\mathbf{P}$ & $815 \pm 149$ & $1.019 \pm 40$ & $662 \pm 110$ & $5,80^{\circ}$ \\
\hline & 0 & $407 \pm 74$ & $306 \pm 31$ & $331 \pm 65$ & $1,00^{\mathrm{NS}}$ \\
\hline & U & $1,75^{*}$ & $2,03^{*}$ & $1,75^{\mathrm{NS}}$ & \\
\hline \multirow{3}{*}{$\begin{array}{l}N^{\circ} \text { promedio } \pm \text { error estándar de individuos } \\
\text { fitófagos/ha }\end{array}$} & $\mathbf{P}$ & $331 \pm 148$ & $331 \pm 148$ & $26 \pm 11$ & $5,10^{\mathrm{NS}}$ \\
\hline & $\mathrm{O}$ & $127 \pm 57$ & $76 \pm 34$ & $0 \pm 0$ & $2,71^{\mathrm{NS}}$ \\
\hline & U & $0,41^{\mathrm{NS}}$ & $1,76^{*}$ & $-0,71^{\mathrm{NS}}$ & \\
\hline \multirow{3}{*}{$\begin{array}{l}N^{\circ} \text { promedio } \pm \text { error estándar de individuos } \\
\text { depredadores/ha }\end{array}$} & $\mathbf{P}$ & $1.553 \pm 211$ & $2.699 \pm 291$ & $3.718 \pm 393$ & $10,00^{* *}$ \\
\hline & 0 & $433 \pm 76$ & $306 \pm 65$ & $306 \pm 65$ & $1,83^{\mathrm{NS}}$ \\
\hline & U & $2,02^{*}$ & $2,02^{*}$ & $2,02^{*}$ & \\
\hline \multirow{3}{*}{$\begin{array}{l}N^{\circ} \text { promedio } \pm \text { error estándar de individuos } \\
\text { saprófagos/ha }\end{array}$} & $P$ & $0 \pm 0$ & $0 \pm 0$ & $306 \pm 137$ & $3,38^{*}$ \\
\hline & 0 & $51 \pm 23$ & $102 \pm 46$ & $127 \pm 57$ & $0,98^{\mathrm{NS}}$ \\
\hline & U & $-2,07^{\mathrm{NS}}$ & $-2,12^{\mathrm{NS}}$ & $0,41^{\mathrm{NS}}$ & \\
\hline
\end{tabular}

\section{DISCUSIÓN}

Este estudio proporciona información que contribuye con el conocimiento del ensamble de coleópteros epigeos ribereños del Humedal de Batuco y de la variación estacional de su abundancia y riqueza y del comportamiento de sus gremios tróficos en función del TV de distinta composición florística. Los coleópteros epigeos más abundantes pertenecieron a Carabidae tanto en primavera $(93,1 \%)$ como en otoño $(81,7 \%)$. Esta familia posee cualidades ecológicas que permiten su uso como modelos de interpretación de fenómenos de intervención antrópica (Uribe \& Vallejo 2013), por lo que ciertas especies potencialmente podrían ser usadas con estos propósitos en un futuro, requiriéndose más estudios. Los carábidos (larvas y adultos) son esencialmente depredadores, situándose en la cima de la pirámide de la cadena trófica, permitiéndoles vivir en ecosistemas generalmente poco apropiados para los demás insectos. La calidad y estructura de la vegetación influyen en los patrones de distribución espacial de los carábidos y afectan a los depredadores mediante la disponibilidad de su alimento (Roig-Juñent \& Domínguez 2001). En este sentido, la mayoría de las especies de carábidos detectadas fueron riparias, hidrófilas o mesófilas con afinidad por los sitios húmedos como Argutoridius chilensis, a las que se añaden especies más xerófilas o subxerófilas como Pachymorphus chalceus y también la especie ubicua y sinantrópica Pachymorphus striatulus (Castro et al. 2012).

Durante la primavera se registró una abundancia y riqueza de coleópteros epigeos significativamente superior en todos los TV estudiados. Cabe destacar que la abundancia en primavera fue notoriamente elevada en los tres TV, aunque en particular, en el TV3 (10 veces superior), seguida del TV2 (seis veces superior que en otoño). En el TV2 la abundancia se podría explicar por la composición de las especies vegetales, asociadas a condiciones de salinidad variable: Chenopodium glaucum (menos halófita), Distichlis spicata (más halófita), y junto a Proustia cuneifolia, de menor humedad edáfica (más xéricas) (Del Campo et al. 2005), que permiten un hábitat 
más heterogéneo para el establecimiento de coleópteros. Si bien la mayor abundancia se obtuvo en el TV3, formada por especies menos halófitas (E. macrostachya, S. californicus y $T$. angustifolia) y con una mayor humedad edáfica (Del Campo et al. 2005) que las encontradas en los TV1 y TV2, ésta se logró principalmente por el aporte de una única especie. También estos incrementos se podrían entender por una mayor disponibilidad de alimento, proveniente de especies vegetales adaptadas a menor salinidad, así como por otros factores que podrían favorecer su establecimiento y sobrevivencia (Grez et al. 2003; Correa \& Roa 2005), tales como refugios (Ramírez et al. 2017; Alvarado-Orellana et al. 2018). La disponibilidad de alimento es un factor determinante en el incremento de la abundancia de insectos, especialmente de los fitófagos, ya que existe una dependencia entre los estadios del ciclo de desarrollo de estos insectos con la vegetación disponible, es decir, una baja calidad de alimento podría significar un retraso en la madurez sexual del individuo, o en las hembras una reducción de la ovulación, lo que generaría una disminución en la disponibilidad de presas para los insectos depredadores, afectando directamente su densidad poblacional (Buddle \& Hammond 2003; Fernández et al. 2014). En particular, con respecto a los carábidos, en un estudio de un agroecosistema bonaerense, tanto el uso del suelo como la estructura de la vegetación se consideró que influyeron en su diversidad (Castro et al. 2017). Por otro lado, la mayor riqueza significativa de coleópteros registrada en el TV2 podría explicarse por las características de la vegetación asociada, aportando con ello variabilidad de alimento para las especies de insectos. Estos resultados de mayor diversidad de coleópteros epigeos en primavera en ambientes nativos, de mayor diversidad en la riqueza de especies vegetales como en su estructura, usando trampas de intercepción, coinciden con los indicados por Ramírez et al. (2017), donde evaluaron tratamientos de residuos vegetales dejados en terreno posterior a una tala rasa de Pinus radiata, residuos eliminados y otros quemados en una zona de ecotono de bosque de hualo (Nothofagus glauca) (Constitución, Región del Maule), siendo el tratamiento de residuos dejados (no intervenidos) que recibieron los efectos de las especies nativas aledañas que se refugiaron, los que presentaron mayor abundancia y riqueza. En el mismo sentido, Alvarado-Orellana et al. (2018), con idéntico método de desarrollado en el presente trabajo, en este caso en los bordes de la Laguna Carén (Santiago, Región Metropolitana) determinaron que las zonas con mayor vegetación y menos antropizadas, tuvieron una mayor diversidad de especies de coleópteros epigeos.

En el TV2 donde se concentró la segunda mayor diferencia en la abundancia de los coleópteros epigeos, se generó también un cambio en la vegetación circundante, fragmentándose. Esta variación se podría explicar por la susceptibilidad de los insectos a la fragmentación de su hábitat, ya que cambia la distribución, diversidad y/o abundancia, en función de la configuración espacial final del paisaje, pudiendo incluso responder frente a fragmentaciones a pequeña escala donde su respuesta puede ser favorable, perjudicarlos o no influir en su abundancia (Rukke 2000; With et al. 2002; Braschler et al. 2003). Se estima que existe una relación positiva entre la riqueza específica de la entomofauna y la heterogeneidad de la vegetación (Villagrán-Mella et al. 2006), por lo cual un incremento del desarrollo antrópico pudiera producir un cambio en la dinámica de los ecosistemas. Esto podría llegar a afectar no sólo a los organismos presentes, sino que también a los cuerpos de agua (Figueroa et al. 2003).

La fragmentación causa que los recursos (e.g. alimento, refugio, parejas) se distribuyan más heterogéneamente, y la estructura y abundancia de las poblaciones están directamente relacionadas con la disponibilidad de recursos (Castiglioni et al. 2017). Las especies que se posicionan en los niveles más altos de la cadena alimenticia, como los depredadores detectados en este estudio, son usualmente más sensibles a la fragmentación que aquellos en los niveles inferiores, como los fitófagos (Hunter 2002). Esta mayor sensibilidad de las especies en niveles tróficos altos a la fragmentación se puede producir porque ésta altera más su capacidad de dispersión que la de sus presas, impidiendo la llegada oportuna a parches aislados (Castiglioni et al. 2017), sin embargo este factor no fue investigado en este estudio por lo que se sugiere incorporarlo en el futuro. Particularmente, se recomienda crear áreas de refugio para promover la diversidad de los coleópteros epigeos, alrededor del cuerpo de agua del humedal, tal como lo señalan Alvarado-Orellana et al. (2018).

Cabe señalar que los diversos factores que pueden incidir en la diversidad de los insectos, en este caso coleópteros, como el tipo de vegetación, aquí estudiado, no pueden ser analizados de forma independiente, sino que se deben integrar, tal como lo indican Sánchez \& Amat (2005), a fin de contribuir a la comprensión de las diferencias de diversidad que se dan en las comunidades de insectos. Por último, esta investigación localizada en el Humedad de Batuco, sitio prioritario, según la Estrategia para la Conservación de la Biodiversidad (CONAMA 2004), se constituye en el primer registro de la fauna de coleópteros epigeos asociada a este ecosistema y aporta al conocimiento de su dinámica estacional, convirtiéndose en la base para estudios futuros enfocados a evaluar el potencial de estos insectos como indicadores del estado de vulnerabilidad de este ecosistema, que recientemente ha sido aprobado para convertirse en Santuario de la Naturaleza (MMA 2021). 


\section{AGRADECIMIENTOS}

Al Fondo de Apoyo para Publicaciones Científicas, del Departamento de Silvicultura y Conservación de la Naturaleza, Universidad de Chile y al Museo Nacional de Historia Natural.

\section{REFERENCIAS}

Alvarado-Orellana, A., Huerta-Fuentes, A., Palma-Muñoz, A., Rodríquez-Tobar, S. 2018. Variación estacional de la diversidad de coleópteros epigeos en la Laguna Carén (Santiago, Chile). Revista Colombiana Entomología 44(2): 266-272.

Artigas, J.N. 1994. Entomología económica. Insectos de interés agrícola, forestal, médico y veterinario (nativos, introducidos y susceptibles de ser introducidos). Ediciones Universidad de Concepción, Concepción, Chile.

Barba-Álvarez, R., De la Lanza-Espino, G., Contreras-Ramos, A., González-Mora, I. 2013. Insectos acuáticos indicadores de calidad del agua en México: casos de estudio, ríos Copalita, Zimatán y Coyula, Oaxaca. Revista Mexicana de Biodiversidad 84(1): 381-383.

Bautista, F., Delfín, H., Palacio, J., Delgado, M. 2011. Técnicas de muestreo para manejadores de recursos naturales. Segunda Edición. Universidad Nacional Autónoma de México. Centro de Investigación en Geografía Ambiental Instituto Nacional, México D.C. 772 pp.

Braschler, B., Lampel, G., Baur, B. 2003. Experimental smallscale grassland fragmentation alters aphid population dynamics. Oikos 100: 581-591.

Briones, R., Jerez, V. 2007. Efecto de la edad de la plantación de Pinus radiata en la abundancia de Ceroglossus chilensis (Coleoptera: Carabidae) en la Región del Biobío, Chile. Bosque 28(3): 207-2014.

Briones, R., Gárate, F., Jerez, V. 2012. Guía de campo: Insectos de Chile nativos, introducidos y con problemas de conservación. Corporación Chilena de la Madera, Concepción, Chile. 257 pp.

Buddle, C.M, Hammond, H.E.J. 2003. Comparison of ground beetles (Coleoptera: Carabidae) and spiders (Araneae) collected in pan and pitfall traps. The Canadian Entomologist 135: 609-611.

Castiglioni, E., García, L.F, Burla, J.P., Arbulo, N., Fagúndez, C. 2017. Arañas y carábidos como potenciales bioindicadores en ambientes con distinto grado de intervención antrópica en el este uruguayo: un estudio preliminar. Revista del Laboratorio Tecnológico de Uruguay 13: 106-114.

Castro, A.V, Porrini, D.P., Cicchino, A.C. 2012. Ensamble peridomiciliario de carábidos (Coleoptera: Carabidae) en un talar del sudeste bonaerense, Argentina. Revista de la Sociedad Entomológica Argentina 71(3-4): 231-247.

Castro, A.V, Porrini, D.P., Cicchino, A.C. 2017. Diversidad de Carabidae (Insecta: Coleoptera) en distintos ambientes de un agroecosistema del sudeste bonaerense, Argentina. Ecología Austral 27(2): 252-265.

Cepeda-Pizarro, J., Pizarro-Araya, J., Vásquez, H. 2005. Variación en la abundancia de Arthropoda en un transecto latitudinal del desierto costero transicional de Chile, con énfasis en los tenebriónidos epigeos. Revista Chilena de Historia Natural 78: 651-663.

CONAMA. 2004. Guía CONAMA para el Establecimiento de las Normas Secundarias de Calidad Ambiental para Aguas Continentales Superficiales y Marinas Comisión Nacional del Medio Ambiente, Santiago, Chile. 18 pp.

CONAF. 2010. Programa nacional para la conservación de humedales insertos en el Sistema Nacional de Áreas Silvestres Protegidas del Estado. CONAF, Convenio de eficiencia institucional, Departamento de Conservación de la Diversidad Biológica, Santiago, Chile. 100 pp.

Correa, P., Roa, A. 2005. Relaciones tróficas entre Oncifelis guigna, Lycalopex culpaeus, Lycalopex griseus y Tyto alba en un ambiente fragmentado de la zona central de Chile. Mastozoología Neotropical 12: 57-60.

Del Campo, P., Luebert F., Teillier, S. 2005. Asociaciones vegetales de la laguna de Batuco. Región Metropolitana. Chile. Chloris Chilensis: Año 8 No 1. http://www.chlorischile.cl. Accedido: Junio 22, 2020.

Donoso, C. 1993. Bosques templados de Chile y Argentina. Variación, Estructura y Dinámica. Editorial Universitaria. Santiago, Chile. 484p.

Elgueta M., Arriagada, G. 1989. Estado actual del conocimiento de los coleópteros de Chile (Insecta: Coleoptera). Revista Chilena de Entomología 17: 5-60.

Fernández, I., Favila, M.E., López, G.M. 2014. Composición, riqueza y abundancia de coleópteros (Coleoptera) asociados a bosques semideciduos y vegetaciones ruderales en la Sierra del Rosario, Cuba. Boletín de la Sociedad Entomológica Aragonesa 54: 329-339.

Figueroa, R., Valdovinos, E., Araya, E., Parra, O. 2003. Macroinvertebrados bentónicos como indicadores de calidad del agua de ríos del sur de Chile. Revista Chilena de Historia Natural 76(2): 275-285.

Grez, A.A., Moreno, P., Elgueta, M. 2003. Coleópteros (Insecta: Coleoptera) epigeos asociados al bosque maulino y plantaciones de pino aledañas. Revista Chilena de Entomología 29: 9-18.

Hunter, M.D. 2002. Landscape structure, habitat fragmentation, and the ecology of insects. Agricultural and Forest Entomology 4: 159-166. 
InfoStat. 2011. Programa InfoStat versión 2011. Grupo InfoStat, FCA, Universidad Nacional de Córdoba, Córdoba, Argentina.

Jerez, V., Zúñiga-Reinoso, Á., Muñoz-Escobar, C., Pizarro-Araya, J. 2015. Acciones y avances sobre la conservación de insectos en Chile. Gayana 79(1): 1-3.

Luebert, F., Pliscoff, P. 2006. Sinopsis Bioclimático y Vegetacional de Chile. Editorial Universitaria, Santiago, Chile. 316 pp.

Martínez, E., Flores, J.P., Poblete, V., Vita, A., Retamal, M., Moya, I. 2011. Sistematización de información para el diagnóstico del estado actual del bosque esclerófilo en ChileBiblioteca digital. Informe Final. Centro de Información de Recursos Naturales. Santiago, Chile. 80 pp.

Mittermeier, R.A., Robles, P., Hoffmann, M., Pilgrim, J., Brooks, T., Mittermeier, C.G., Lamoreux, J., Da Fonseca, G.A. 2004. Hotspots, Biodiversidad amenazada II: Nuevas ecorregiones terrestres prioritarias del mundo. Agrupación Sierra Madre, Editorial Cemex, México D.C. 390 pp.

Morrone, J.J. 1993. Revisión sistemática del género Hyperoides Marshall. Neotrópica 39: 17-26.

Moreno, C.E. 2001. Métodos para medir la biodiversidad. M\&T Manuales y Tesis SEA. Vol. I. Zaragoza, España. 84 pp.

MMA. 2018. Biodiversidad de Chile. Patrimonio y Desafíos. Tercera Edición. Tomo I. Ministerio del Medio Ambiente, Santiago, Chile. 480 pp.

MMA. 2021. Laguna Batuco es declarada Santuario de la Naturaleza por el consejo de ministros para la sustentabilidad. Minissterio del Medio Ambiente, Chile. URL: https://mma.gob.cl/laguna-de-batuco-es-declaradasantuario-de-la-naturaleza-por-el-consejo-de-ministrospara-la-sustentabilidad Accedido: Junio 10, 2021.

Myers, N., Mittermeier, R.A., Mittermeier, C.G., Da Fonseca, G.A., Kent, J. 2000. Biodiversity hotspots for conservation priorities. Nature 403: 853-858.

Ramírez, P., Huerta, A., Araya, J.E. 2017. Effect of post-harvest forestry residue management practices on the diversity of epigeal coleopterans. Revista Facultad Nacional de Agronomía 70(1): 8069-8075.
Roig-Juñent, S., Domínguez, M.C. 2001. Diversidad de la familia Carabidae (Coleoptera) en Chile. Revista Chilena de Historia Natural 74: 549-571.

Rukke, B.A. 2000. Effects of habitat fragmentation: increased isolation and reduced habitat size reduces the incidence of dead wood fungi beetles in a fragmented forest landscape. Ecography 23: 492-502.

Sala, O.E., Chapin, F.S., Armesto, J.J., Berlow, E.L., Bloomfield, J.B., Dirzo, R.H., Huber-Sanwald, E., Huenneke, L., Jackson, R.B., Kinzig, A.P., Leemans, R., Lodge, M.L., Mooney, H.A., Oesterheld, M., Poff, N.L., Sykes, M.T., Walter, B., Walter, M., Wall, D.H. 2000. Biodiversity - Global biodiversity scenarios for the year 2100. Science 287: 1770-1774.

Sánchez, D., Amat, G.D. 2005. Diversidad de la fauna de artrópodos terrestres en el Humedal Jaboque, Bogotá, Colombia. Caldasia 27(2): 311-329.

Uribe, J.M., Cabrera, R., De la Fuente, A., Paneque, M. 2012. Atlas Bioclimático de Chile. CORFO: Ministerio de Bienes Nacionales, Santiago, Chile. 228 pp.

Uribe, M., Vallejo, L.F. 2013. Diversidad de escarabajos Carabidae y Scarabaeidae de un bosque tropical en el Magdalena Medio Colombiano. Boletín Científico Centro de Museos de Historia Natural 17(2): 174-196.

Vásquez, G., Castro, G., González, I, Pérez, R., Castro, T. 2006. Bioindicadores como herramientas para determinar la calidad del agua. Revista Contactos 60: 41-48.

Villagrán-Mella, R., Aguayo, M., Parra, L.E., González, A. 2006. Relación entre características del hábitat y estructura del ensamble de insectos en humedales palustres urbanos del centro-sur de Chile. Revista Chilena de Historia Natural 79: 195-211.

Werner, F.G. 1974. A review of the Chilean Anthicidae (Coleoptera). Revista Chilena de Entomología 8: 27-32.

With, K.A., Pavuk, D.M., Worchuck, J.L., Oates, R.K., Fisher, J.L. 2002. Threshold effects of landscape structure on biological control in agroecosystems. Ecological Applications 12: 52-65. 